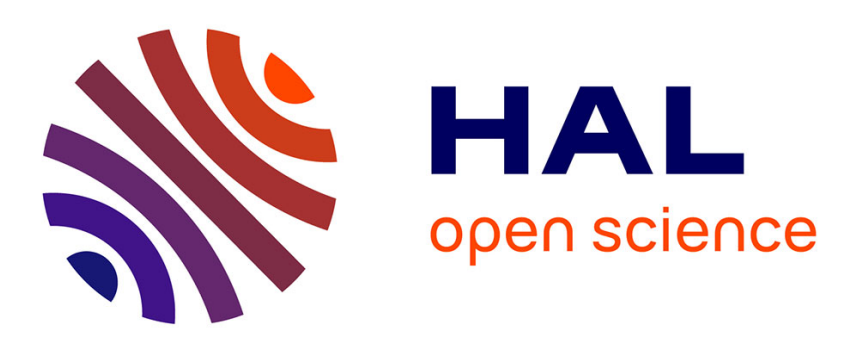

\title{
Embedding information on communicating materials from context-sensitive information analysis based on Fuzzy AHP theory
}

\author{
Sylvain Kubler, William Derigent, Eric Rondeau, André Thomas
}

\section{To cite this version:}

Sylvain Kubler, William Derigent, Eric Rondeau, André Thomas. Embedding information on communicating materials from context-sensitive information analysis based on Fuzzy AHP theory. IEEE International Conference on Internet of Things, Nov 2012, Besançon, France. pp.240-243. hal00756546

\section{HAL Id: hal-00756546 \\ https://hal.science/hal-00756546}

Submitted on 26 Nov 2012

HAL is a multi-disciplinary open access archive for the deposit and dissemination of scientific research documents, whether they are published or not. The documents may come from teaching and research institutions in France or abroad, or from public or private research centers.
L'archive ouverte pluridisciplinaire HAL, est destinée au dépôt et à la diffusion de documents scientifiques de niveau recherche, publiés ou non, émanant des établissements d'enseignement et de recherche français ou étrangers, des laboratoires publics ou privés. 


\title{
Embedding information on communicating materials from context-sensitive information analysis based on Fuzzy AHP theory
}

\author{
Sylvain Kubler and William Derigent and Éric Rondeau and André Thomas \\ Centre de Recherche en Automatique de Nancy, Université de Lorraine, CNRS UMR 7039 \\ Campus Sciences, BP 70239, F-54506 Vandouvre-lès-Nancy Cedex, France, \\ Sylvain.Kubler@cran.uhp-nancy.fr
}

\begin{abstract}
The idea of using intelligent products to ensure an information continuum all along the product life cycle (PLC) is more and more shared today. However, it is not that easy to identify what information should be stored on the product. To answer this question, we propose a data dissemination process to select context-sensitive information from the database, that must be stored/replicated on the product. Our approach uses the fuzzy-AHP theory for aggregating points of view from different actors.
\end{abstract}

Keywords-Product Lifecycle Management; Data Dissemination; Fuzzy AHP; Pervasive Computing

\section{INTRODUCTION}

New challenges and opportunities arise with concepts such as internet of things (IoT), artificial intelligence, or still ubiquitous computing [1]. The idea of using intelligent products to ensure an information continuum all along the product life cycle (PLC) is more and more shared today [2]. Främling et al. [3] argue that it is a formidable challenge to link the product related information to the products themselves, making the information of all the product components easily achievable. To do so, several solutions and platforms have emerged such as EPCglobal, ID@URI or WWAI [4]. However, products are only given an identifier (e.g. via a RFID tag) which provides a network pointer to a linked database; these solutions do not answer the questions of (i) what information is relevant to users? and (ii) where information should be stored? (on the product or on the database) [5].

As a result, we propose a data dissemination process in two steps to disseminate information between databases and communicating products:

- Process step 1 aims at selecting, at a given moment of the PLC, relevant information from the database that should be stored on the product,

- Once data that must be stored on the product is identified, it is necessary to develop tools to write/read this data on/from the product. This is achieved via process step 2 which was the subject of previous work [6],

This paper takes focus on the process step 1 and an approach is proposed in section $\amalg$ and $\amalg$ to select contextsensitive information (from the database), that should be stored/replicated on the product. Our approach uses the fuzzy-AHP theory for aggregating multiple points of view.

\section{TOWARDS THE USE OF THE FUZZY-AHP THEORY}

This paper develops an approach to select contextsensitive information. This approach takes as input the
Logical Data Model (LDM). Figure 1 gives insight into a part of such a LDM. One entity from the LDM corresponds to a relational table as depicted in Figure 1 (entity/table: Material), where the attributes listed in each entity correspond to the table columns and, each table row is referred to as a tuple/instance of the relation. Our approach consists in assessing the relevance of storing a given "data item" (i.e. one table cell) on the product. Figure 1 gives the relevance value of all data items from the table Material (e.g. the relevance of the data item located at row 3 , column 1 , noted $\mathrm{T}_{\mathrm{MD}\{3,1\}}$, is equal to 0.2 ). The higher the relevance value, the higher the probability that the data item will be stored on the product. Finally, data items can therefore be classified in order of relevancy. In the PLC, several experts are concerned by selecting data that must be stored on the product, therefore generating different points of view. All points of view are legitimate and must be taken into account. Accordingly, this paper proposes an approach which combines the use of the analytic hierarchy process - AHP (to structure the multi-criteria decision making problem - MCDM) [7] with the fuzzy logic (to express and aggregate multiple points of view). [8] introduced a new approach for handling fuzzyAHP, with the use of triangular fuzzy numbers for pairwise comparison scale and the use of the extent analysis method (FEAHP).

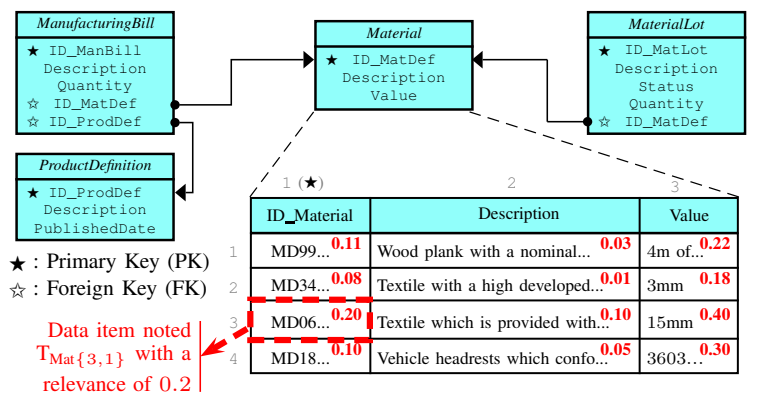

Figure 1. View of a Logical Data Model (LDM) and a relational table

\section{DATA RELEVANCE COMPUTATION USING FEAHP}

In a classical set $\mathcal{A}$, an element belongs entirely or not to $\mathcal{A}$. In a fuzzy set $\tilde{\mathcal{A}}$, an element can have different degrees of membership to $\tilde{\mathcal{A}}$. For each element of the referential $x$, a degree of membership to the fuzzy set $\tilde{\mathcal{A}}$ is assigned, noted $\mu_{\tilde{\mathcal{A}}}(x)$. Thus, when $\mu_{\tilde{\mathcal{A}}}(x)=1, x$ belongs entirely to $\tilde{\mathcal{A}}$; when $\left.\left.\mu_{\tilde{\mathcal{A}}}(x) \in\right] 0 ; 1\right], x$ belongs more or 
less to $\tilde{\mathcal{A}}$. FEAHP uses triangular fuzzy numbers, noted $[L, M, U]$ (see Figure 5) and consists of five stages as depicted in Figure 2 Each stage is described and detailed in the five next sections.

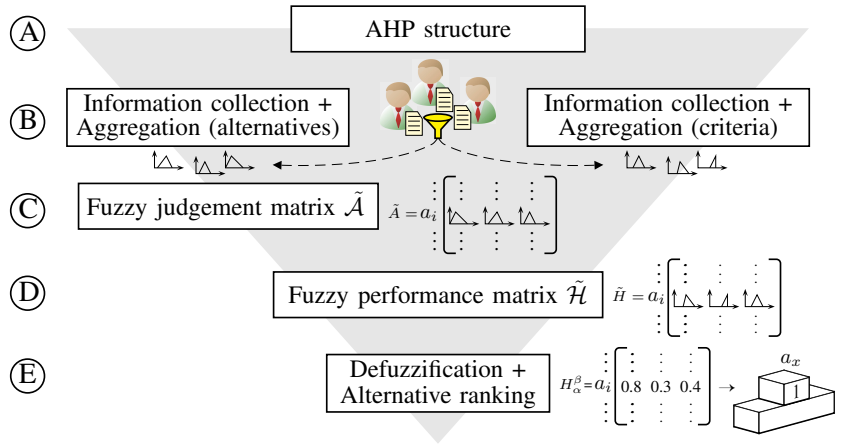

Figure 2. Fuzzy AHP consisting of 5 stages

\section{A. AHP structure}

Our MCDM problem is broken down into the hierarchical structure depicted in Figure 3 . The alternatives are the data items ( $c f$. level 3) which must be assessed and ranked in term of relevancy ( $c f$. level 1$)$. Three criteria are defined at level 2: Enumeration, Contextual and Data Size which are respectively abbreviated $\mathrm{C}_{e}, \mathrm{C}_{c}, \mathrm{C}_{s}$ and are detailed in the next stage/section.

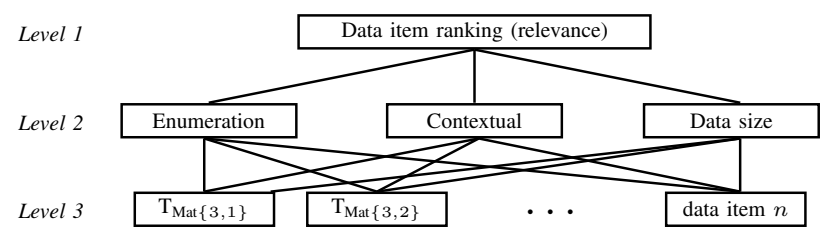

Figure 3. General architecture of the hierarchy

\section{B. Information collection + Aggregation}

In this section, we provide information about the way to evaluate each alternative with respect to each criterion $\left(\mathrm{C}_{e}\right.$, $\mathrm{C}_{c}, \mathrm{C}_{m}$ ) and the way to obtain the criteria importance.

1) $C_{e}$ : Through this criterion, the user may enumerate information (s)he judges important to store on the product. To do so, each decision maker $p$ enumerates attributes from tables. Let $t$ a table of $\mathcal{T}$ and $v$ an attribute of $t$. If the attribute $v$ is enumerated by $p$, the enumeration score $s^{p}(v, t)=1$, otherwise 0 , as noted in equation 1 . Then, all decision makers' opinion $s^{p}(v, t)$ are aggregated in equation 2 through a triangular fuzzy number $\tilde{s}(v, t)$. Let us remind ourselves that an attribute corresponds to a column of a table, which in turn, consists of one or many data items. As a result, if a data item $l$ belongs to $v$, its score with respect to the criterion $\mathrm{C}_{e}$, noted $\tilde{\phi}_{e}(l)$, will therefore be equal to $\tilde{s}(v, t)$.

$$
\begin{aligned}
& s^{p}(v, t)=\left\{\begin{array}{ll}
1 & \text { enumerated } \\
0 & \text { not enumerated }
\end{array}, \forall p=\left\{1 . . p_{\max }\right\}\right. \\
& \tilde{s}(v, t)=\left[\begin{array}{ccc}
L & M & U \\
\min \left(s^{p}(v)\right) & \frac{\sum_{p=1}^{p_{\max }} s^{p}(v)}{p_{\max }} & \left.\max \left(s^{p}(v)\right)\right]
\end{array}\right.
\end{aligned}
$$

2) $C_{c}$ : The previous criterion allows users to specify information that must be stored on the product. However, they could omit important information. Indeed, they might not be aware of all the data needed by the downstream actors (along the PLC). As a result, a new criterion referred to as contextual $\left(\mathrm{C}_{c}\right)$ is integrated in order to moderate and to balance $\mathrm{C}_{e}$. First, let us note that a multitude of information systems exist over the PLC (e.g. ERP, PDM, MES) which are not concerned by the same data (i.e. the same entities from the LDM). The idea is to identify specific "entity groups" through the LDM according to, for instance, the information systems and, therefore, to evaluate their importance over the PLC. In our study, each decision maker $p$ performs pairwise comparisons between entity groups as in equation 3 with $z$ the number of groups defined trough the LDM. The importance of entity group $i$ over entity group $j$ evaluated by the decision maker $p$ is noted $s_{i j}^{p}$. This evaluation is based on the 1 to 9 -point scale from [7]: $\{1,3,5,7,9\} . s_{i j}^{p}=1$ means that groups $i$ and $j$ are equal in importance and $s_{p_{i j}}=9$ means that group $i$ is strongly favored over $j$. Then, all score $s_{i j}^{p}$ are aggregated through a triangular fuzzy number, noted $\tilde{s}_{i j}$, in equation 4. Equation 5 is finally applied in order to acquire a unique score/triangular fuzzy number $\tilde{\phi}_{c}\left(\mathrm{G}_{i}\right)$ for each entity group $i . \tilde{\phi}_{c}\left(\mathrm{G}_{i}\right)$ actually indicates the relative importance of information involves in $\mathrm{G}_{i}$. If a data item $l$ is contained in a table included in $\mathrm{G}_{i}$, its score with respect to $\mathrm{C}_{c}$, noted $\tilde{\phi}_{c}(l)$, is therefore equal to $\tilde{\phi}_{G}\left(\mathrm{G}_{i}\right)$.

$$
\begin{gathered}
\mathrm{G}_{1}\left[\begin{array}{ccc}
\mathrm{G}_{1} & \cdots & \mathrm{G}_{z} \\
\vdots & \cdots & s_{1 z}^{p} \\
\vdots & \ddots & \vdots \\
\mathrm{G}_{z} & \cdots & 1
\end{array}\right], s_{j i}^{p}= \begin{cases}s_{i j}^{p} & i=j \\
\left(s_{i j}^{p}\right)^{-1} & i \neq j\end{cases} \\
\tilde{s}_{i j}=\left[\begin{array}{lll}
L & M & M \\
\min \left(s_{i j}^{p}\right) & \frac{\sum_{p=1}^{p_{\max }} s_{i j}^{p}}{p_{\max }} & \max \left(s_{i j}^{p}\right)
\end{array}\right] \\
\tilde{\phi}_{c}\left(\mathrm{G}_{i}\right)=\frac{\sum_{j=1}^{z} \tilde{s}(i, j)}{\sum_{k=1}^{z} \sum_{j=1}^{z} \tilde{s}(k, j)}
\end{gathered}
$$

3) $C_{s}$ : This criterion favors the storage of information on the product according to the data size. Since products are often memory-constrained, data relevance should decrease when data size increases. Such a behavior can be obtained via equation 6 , with $d$ the size of a data item $l$ and $k^{p}$ a constant adjusted by the expert $p$. Figure 4 shows two functions according to the data size $d$ and for two different $k^{p}$. It can be observed that the smaller $k^{p}$ is, the bigger the data authorized to be stored on the product is (e.g., data with sizes $>60$ bytes are neglected when $k=1.08)$.

$$
\left(k^{p}\right)^{-d} \quad k^{p} \in \mathbb{R}_{+} ; d \in \mathbb{N}_{+}
$$

The coefficient $k^{p}$ must be adjusted by each decision maker $p$ as in equation 7 Then, all coefficients $s_{k}^{p}$ are aggregated through a triangular fuzzy number, noted $\tilde{s}_{k}$, in equation 8. The score of an alternative $l$ with respect to $\mathrm{C}_{c}$, noted $\tilde{\phi}_{c}(l)$, is therefore computed in equation 9 with $d$ the size of the data item $l$ in bytes. 


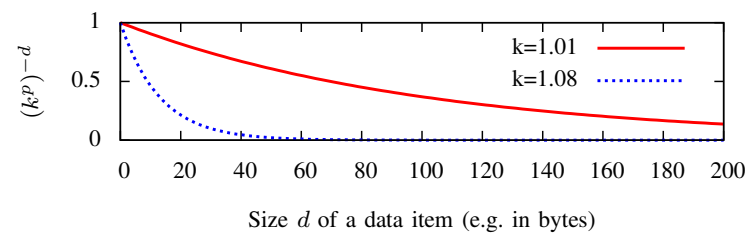

Figure 4. Adjustment of $k^{p}$ performed by a decision maker $p$

$$
\begin{aligned}
& s_{k}^{p}=k^{p} \\
& \tilde{s}_{k}=\left[\begin{array}{ccc}
L & M & U \\
\min \left(s_{k}^{p}\right) & \frac{\sum_{p=1}^{p_{\max }} s_{k}^{p}}{p_{\max }} & \max \left(s_{k}^{p}\right)
\end{array}\right] \\
& \tilde{\phi}_{s}(l)=\left[\begin{array}{lll}
\tilde{s}_{k}(U)^{-d} & \tilde{s}_{k}(M)^{-d} & \tilde{s}_{k}(L)^{-d}
\end{array}\right]
\end{aligned}
$$

4) Criteria importance: Experts specify the criteria importance via pairwise comparisons as in $\mathrm{C}_{c}$. The relative importance of each criterion, noted $\tilde{\phi}\left(\mathbf{C}_{x}\right)$ (see equation (10), is computed based on the same equations than previously (i.e. equations 3 to 5 .

$$
\tilde{\phi}_{x}=\frac{\sum_{j=\left\{\mathrm{C}_{e}, \mathrm{C}_{m}, \mathrm{C}_{s}\right\}} \tilde{s}(x, j)}{\sum_{k=\left\{\mathrm{C}_{e}, \mathrm{C}_{m}, \mathrm{C}_{s}\right\}} \sum_{j=\left\{\mathrm{C}_{e}, \mathrm{C}_{m}, \mathrm{C}_{s}\right\}} \tilde{s}(k, j)}
$$

\section{Fuzzy judgment matrix $\tilde{A}$}

After getting all alternative scores with respect to all criteria, we form the fuzzy judgment matrix $\tilde{A}$ as in equation 11 where $\tilde{\phi}_{x}(l)$ denotes the judgement score of alternative $l$ with respect to criterion $x$.

$$
\tilde{A}=\begin{gathered}
A_{1} \\
\vdots \\
A_{n}
\end{gathered}\left[\begin{array}{cccc}
\tilde{\phi}_{e}(1) & \tilde{\phi}_{c}(1) & \tilde{\phi}_{m}(1) & \tilde{\phi}_{s}(1) \\
\vdots & \vdots & \vdots & \vdots \\
\tilde{\phi}_{e}(n) & \tilde{\phi}_{c}(n) & \tilde{\phi}_{m}(n) & \tilde{\phi}_{s}(n)
\end{array}\right]
$$

\section{Fuzzy Performance Matrix $\tilde{H}$}

At this stage, only scores of alternatives with respect to criteria are taken into account, without considering the relative importance of criteria. As a result, we synthesize the fuzzy matrix $\tilde{A}$ with the criteria importance in a fuzzy performance matrix $\tilde{H}$. The performance score $\tilde{h}_{x}(l)$ consists in multiplying the fuzzy set $\tilde{\phi}_{x}(l)$ by the criterion importance itself $\tilde{\phi}_{x}$ as in equation 12 Finally, the fuzzy performance matrix $\tilde{H}$ is obtained as depicted in Figure 13

$$
\begin{aligned}
& \tilde{h}_{x}(l)=\tilde{\phi}_{x}(l) \times \tilde{\phi}_{x} \\
& \begin{array}{llll}
\mathrm{C}_{e} & \mathrm{C}_{c} & \mathrm{C}_{m} & \mathrm{C}_{s}
\end{array} \\
& \tilde{H}=\vdots \begin{array}{cccc}
A_{1} \\
A_{n}
\end{array}\left[\begin{array}{cccc}
\tilde{h}_{1 e} & \tilde{h}_{1 c} & \tilde{h}_{1 m} & \tilde{h}_{1 s} \\
\vdots & \vdots & \ddots & \vdots \\
\tilde{h}_{n e} & \tilde{h}_{n c} & \tilde{h}_{n m} & \tilde{h}_{n s}
\end{array}\right]
\end{aligned}
$$

\section{E. Defuzzification and alternative ranking}

This stage aims at ranking alternatives according to their fuzzy sets. To do so, defuzzification is first applied to transform each fuzzy set into a crisp value. Then, alternatives will be ranked by considering the crisp values based on the TOPSIS method. Defuzzification and TOPSIS methods are respectively presented in what follows.
1) Defuzzification step: Defuzzification is executed (i) by using the $\alpha$-cut method on the fuzzy performance matrix $\tilde{H}$ and (ii) by taking into account the risk index $\beta$ to compute the crisp performance matrix $H_{\beta}^{\alpha}$.

i. The $\alpha$-cut level of an alternative $l$, noted $h_{l x}^{\alpha}$, is computed via equations 14 to $16 . h_{l x a}^{\alpha}$ and $h_{l x a}^{\alpha}$ denotes respectively the left and right point of the level of the triangle as shown in Figure $5(0 \leq \alpha \leq 1)$. The $\alpha$-cut value reflects the degree of confidence of the decision makers when they subjectively evaluate alternative and criteria scores for the MCDM problem. If the decision makers set up a high degree of confidence, it means that they have gathered enough knowledge to support their decisions.

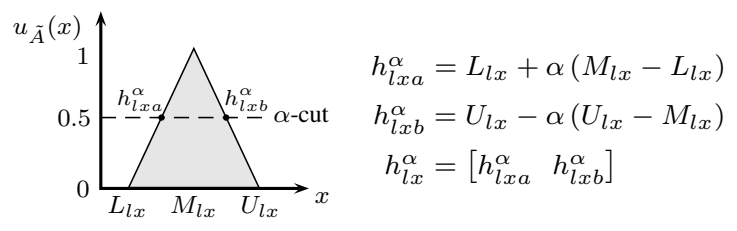

Figure 5. $\alpha$-cut performed on each fuzzy performance score $\tilde{h}_{l x}$

ii. In our approach, the risk index $\beta$ is applied as a defuzzifier. The crisp performance matrix $H_{\beta}^{\alpha}$ is calculated in equation 18 based on equation 17, where $h_{l x \beta}^{\alpha}$ denotes the crisp performance score of alternative $l$ with respect to the criterion $C_{x}$ under a degree of confidence $\alpha$ and a risk level $\beta$. In equation 17 , when $\beta$ continuously increases, the crisp performance score progressively approaches the left point of the interval. A risk index $\beta=\{0,0.5,1\}$ respectively indicates an optimistic, moderate and pessimistic viewpoint of the decision maker choice.

$$
\begin{aligned}
& h_{l x \beta}^{\alpha}=\beta h_{l x a}^{\alpha}+(1-\beta) h_{l x b}^{\alpha} \\
& \begin{array}{llll}
\mathrm{C}_{e} & \mathrm{C}_{c} & \mathrm{C}_{m} & \mathrm{C}_{s}
\end{array}
\end{aligned}
$$

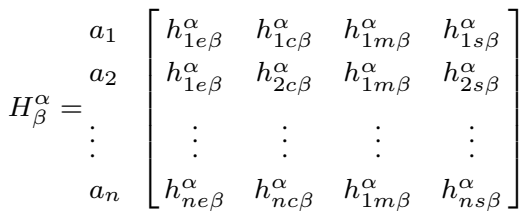

2) Alternative ranking: In TOPSIS, the ideal solution $h_{x \beta}^{\alpha+}$ and the negative ideal solution $h_{x \beta}^{\alpha-}$ are respectively defined as the best and the worst crisp performance scores among all alternatives on a criterion, as specified in equation 19. Subsequently, the distance between the ideal and the negative ideal solution for each alternative is respectively calculated in equations 20 and 21, where $S_{i \beta}^{\alpha+}$ and $S_{l \beta}^{\alpha-}$ represent the distance between the crisp performance scores $h_{l x \beta}^{\alpha}$ of an alternative $l$ with respect to all criteria $\mathrm{C}_{x}$ and all the ideal and negative ideal solutions $h_{x \beta}^{\alpha+}, h_{x \beta}^{\alpha-}$, respectively.

$$
\begin{aligned}
h_{x \beta}^{\alpha+} & =\max _{l}\left(h_{l x \beta}^{\alpha}\right) \quad h_{x \beta}^{\alpha+}=\min _{l}\left(h_{l x \beta}^{\alpha}\right) \quad \forall l=1 . . n \\
S_{l \beta}^{\alpha+} & =\sqrt{\sum_{x}\left(h_{l x \beta}^{\alpha}-h_{x \beta}^{\alpha+}\right)^{2}} \quad l=1,2, . ., n \\
S_{l \beta}^{\alpha-} & =\sqrt{\sum_{x}\left(h_{l x \beta}^{\alpha}-h_{x \beta}^{\alpha-}\right)^{2}} \quad l=1,2, . ., n
\end{aligned}
$$




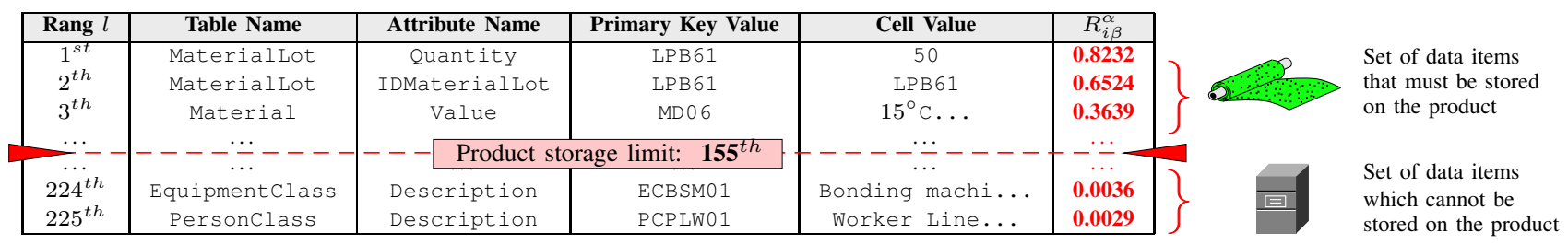

Figure 6. List of data items ordered from the highest relevant to the smallest: $R_{l \beta}^{\alpha}$ indicating the relevance of data item $l$

A prior alternative has a longer distance to the negative ideal solution and a shorter distance to the ideal solution. Consequently, the relative closeness to the ideal solution for each alternative can be formulated as in equation 22 , where $R_{l \beta}^{\alpha}$ denotes the final performance score. The larger the score $R_{l \beta}^{\alpha}$, the more relevant the alternative $l$. All alternatives/data items can therefore be ranked from the more relevant to the less relevant.

$$
R_{l \beta}^{\alpha}=\frac{S_{l \beta}^{\alpha-}}{S_{l \beta}^{\alpha+}+S_{l \beta}^{\alpha-}} \quad l=1,2, . ., n
$$

\section{CASE STUDY}

In this section, a specific scenario is considered in which 225 data items are assessed (data items coming from a LDM of 19 entities). Results about their relevance and ranking are commented hereafter.

Figure 6 provides the resulting list of data items ordered from the highest relevant (see $R_{l \beta}^{\alpha}$ ) to the smallest. The two more relevant data items come from MaterialLot (from attributes Quantity and IDMaterialLot) and the $3^{\text {rd }}$ is $\mathrm{T}_{\mathrm{MD}\{3,3\}}$ (cf. Figure 1).

In our scenario, the 19 entities are clustered in 4 entity groups. The Equipment and Personal data groups, noted respectively $G_{1}$ and $G_{2}$, report information about equipments and persons which/who are somehow related to the product (e.g. equipments used for manufacturing it). The Product and Production data groups, noted respectively $\mathrm{G}_{3}$ and $\mathrm{G}_{4}$, relate respectively information about the product composition (e.g. raw material) and operations (e.g. production rule). In our scenario, adjustments made by experts concerned by the contextual criterion indicates that information included in $\mathrm{G}_{3}$ is of the highest importance comparatively to the other groups. Moreover, the experts indicate that this criterion $\left(\mathrm{C}_{c}\right)$ is one of the most important at this stage of the PLC, with $\mathrm{C}_{e}$. No more than the first 155 data items from the list can be embedded on the product because its memory is limited (highlighted in Figure 6). The pie chart in Figure 7 shows the percentage of data items among the first 155 which belong to each entity group. We can observe that $49 \%$ (i.e. $\approx 75$ data items) are included in tables clustered in $\mathrm{G}_{4}$ and $38 \%$ in tables clustered in $\mathrm{G}_{3}$. This is largely due to the choices made by the experts. Finally, the first 155 data items from the list will be stored on the product thanks to process step 2 ( $c f$. section \).

\section{CONClusion}

The idea of using intelligent products to ensure an information continuum all along the product life cycle

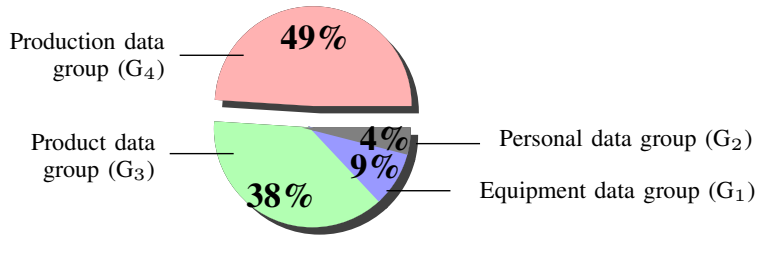

Figure 7. Result of data item relevance in form of a pie chart

(PLC) is more and more shared today. However, it is not that easy to identify the information that must be linked to the product. As a result, this paper proposes an information dissemination process for selecting information sensitive to the context of use of the product. This information is then stored on the products themselves using a new type of augmented material, referred to as "communicating material". The approach developed in this paper uses the fuzzy-AHP theory for aggregating multiple expert's points of view on the product, which are all legitimate.

\section{REFERENCES}

[1] M. Weiser, "The computer for the 21st century," Scientific American, vol. 265, no. 3, pp. 94-104, 1991.

[2] C. Y. Wong, D. Mcfarlane, A. A. Zaharudin, and V. Agarwal, "The Intelligent Product Driven Supply Chain," in International Conference on Systems, Man and Cybernetics, 2002, pp. 4-6.

[3] K. Främling, T. Ala-Risku, M. Kärkkäinen, and J. Holmström, "Agent-based model for managing composite product information," Computers in Industry, vol. 57, no. 1, pp. 7281,2006

[4] G. Meyer, K. Främling, and J. Holmström, "Intelligent products: A survey," Computers in Industry, vol. 60, no. 3, pp. 137-148, 2009.

[5] D. McFarlane, V. Giannikas, A. C. Wong, and M. Harrison, "Intelligent products in the supply chain - 10 years on," in 14th Symposium of Information Control Problems in Manufacturing, 2012.

[6] S. Kubler, W. Derigent, A. Thomas, and É. Rondeau, "Storage of information on manufactured products using "communicating materials"," in AI Problems and Approaches for Intelligent Environments (AI@IE), 2012.

[7] T. Saaty, The Analytic Hierarchy Process. New York: McGraw-Hill, 1980.

[8] D. Chang, "Applications of the extent analysis method on fuzzy ahp," European journal of operational research, vol. 95, no. 3, pp. 649-655, 1996. 